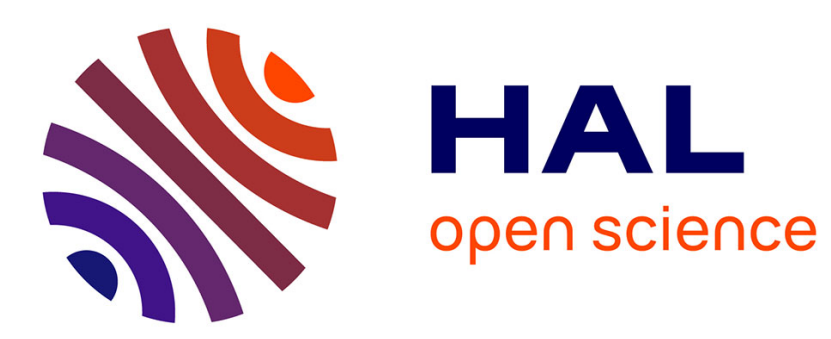

\title{
Conjectures on the transition from Poiseuille to plug flow in suspensions
}

\author{
P.-G. de Gennes
}

\section{To cite this version:}

P.-G. de Gennes. Conjectures on the transition from Poiseuille to plug flow in suspensions. Journal de Physique, 1979, 40 (8), pp.783-787. 10.1051/jphys:01979004008078300 . jpa-00209163

\section{HAL Id: jpa-00209163 https://hal.science/jpa-00209163}

Submitted on 1 Jan 1979

HAL is a multi-disciplinary open access archive for the deposit and dissemination of scientific research documents, whether they are published or not. The documents may come from teaching and research institutions in France or abroad, or from public or private research centers.
L'archive ouverte pluridisciplinaire HAL, est destinée au dépôt et à la diffusion de documents scientifiques de niveau recherche, publiés ou non, émanant des établissements d'enseignement et de recherche français ou étrangers, des laboratoires publics ou privés. 


\title{
Conjectures on the transition from Poiseuille to plug flow in suspensions
}

\author{
P. G. de Gennes \\ Collège de France, 75231 Paris Cedex 05, France \\ (Reçu le 24 janvier 1979, révisé le 4 avril 1979, accepté le 23 avril 1979)
}

\begin{abstract}
Résumé. - Un système de sphères dures (assez grosses pour que le mouvement Brownien soit négligeable) est suspendu dans un fluide visqueux, et un cisaillement macroscopique $s$ est imposé. Dans cette situation, en régime stationnaire, la probabilité $p$ pour une sphère d'être en contact avec ses voisines est finie $(p>0)$ et indépendante de $s$. Quand la fraction en volume $\varphi$ des sphères augmente, elles s'associent progressivement en amas. Nous postulons que pour $\varphi$ supérieure à une valeur critique $\varphi_{c}$, un amas infini apparaît - en analogie avec la percolation. L'hydrodynamique est décrite alors par deux champs de vitesse, et conduit assez naturellement aux écoulements bouchon observés en pratique. Mais le modèle prédit une anomalie de la viscosité apparente en fonction de $\varphi$ qui ne paraît pas avoir été observée.
\end{abstract}

\begin{abstract}
A system of hard spheres (with negligible Brownian motion) is suspended in a viscous fluid, and a macroscopic shear rate $s$ is imposed. In the resulting steady state, the probability $p$ for one sphere to be exactly in contact with another sphere is finite $(p>0)$ and independent of $s$. When the concentration $\varphi$ of spheres increases, they progressively become associated in clusters. We postulate that, when $\varphi$ exceeds a certain critical value $\varphi_{c}$, an infinite cluster appears, in analogy with percolation problems. The hydrodynamics must then include two macroscopic velocity fields, and leads naturally to plug flows, in qualitative agreement with experimental observations; however, the model predicts an anomaly in the plot of apparent viscosity versus concentration, which has not yet been observed.
\end{abstract}

1. Plug flows. - Flows of solid suspensions in long tubes, at low Reynolds numbers have been studied with great care [1]. Here we shall restrict our attention to suspensions of spheres, with exactly adjusted densities and without any attraction between the particles. The sphere radius $(a)$ is chosen large enough to make Brownian motion negligible; explicitly this implies $s \tau \gg 1$, where $s$ is the shear rate, and $\tau=8 \pi a^{3} \eta_{0} / k T$ a diffusion time. ( $\eta_{0}$ is the viscosity of the pure liquid, $T$ the temperature, $k$ the Boltzmann constant.) On the other hand, we assume $D \gg a$, where $D$ is the tube diameter, so that a continuum description may be attempted.

Experimentally [1] when the volume fraction $\varphi$ occupied by the spheres exceeds a certain limit $\varphi_{c}$ (of order $1 / 5$ ) the velocity profile shows a flat portion (plug) in the centre of the tube; but the whole profile is still linear in the pressure head. In the present note, we construct a tentative model for this remarkable effect, based on an analogy with percolation problems [2].
2. Cluster formation. - The equations of motions for the positions of the various spheres $\left(\mathbf{r}_{i}\right)$ may be taken in the purely viscous form :

$$
\frac{\mathrm{d} \mathbf{r}_{i}}{\mathrm{~d} t}=\mathbf{v}_{0}\left(\mathbf{r}_{i}\right)+\sum_{j} \delta_{i j} \mathbf{f}_{j}
$$

Here $v_{0}(\mathbf{r})$ is the unperturbed velocity field of the matrix $; \mathbf{f}_{j}=-\partial U / \partial \mathbf{r}_{j}$ is the force on sphere $(j)$, $U$ being the total interaction energy of the spheres. (In practice we shall mainly be concerned with hard core interactions.) The coefficient $S_{i i}=\left(6 \pi \eta_{0} a\right)^{-1}$ represents the mobility of one sphere, and $S_{i j}(i \neq j)$ is a tensor describing the drift of sphere $(i)$ in the backflow field created by sphere $(j)$. For large intervals $r_{i j}$ and dilute spheres, $\delta_{i j}$ has the simple form :

$$
\delta_{i j}^{\alpha \beta}=\left[8 \pi \eta_{0} r_{i j}\right]^{-1}\left[\delta_{\alpha \beta}+\frac{r_{i j}^{\alpha} r_{i j}^{\beta}}{r_{i j}^{2}}\right] \text {. }
$$

More detailed equations, incorporating sphere rotation, screening effects and interactions through 
higher multipoles, have been discussed by various authors [3]. Here we shall only make use of certain general features common to eq. (1) and to the more refined theories.

Since Brownian motion is negligible, the spheres will not be described by anything similar to a thermodynamic ensemble. But, under the action of the shearing field [4],

$$
v_{o x}=s y, \quad v_{o y}=v_{o z}=0,
$$

we postulate that the trajectories derived from eq. (1) have the properties of ergodicity and mixing. Then there will exist a well defined dynamical steady state, and all time independent observables must achieve average values which are independent of the magnitude of $s$. (A change of $s$ is equivalent to a change in time units in eq. (1).)

Let us now restrict our attention to hard spheres. In thermal equilibrium, each sphere is in contact with other spheres only during infinitely brief collisions. But in our system, the situation is completely different. Two spheres (1) and (2), drifting in their local velocity fields $\left(\mathbf{v}\left(\mathbf{r}_{1}\right)\right.$ and $\left.\mathbf{v}\left(\mathbf{r}_{2}\right)\right)$ may hit each other, and will remain in contact whenever the ambient velocity field tends to decrease $r_{12}$ below the allowed minimum $(2 a)$. Explicitly, this corresponds to the condition :

$$
\left(\mathbf{r}_{2}-\mathbf{r}_{1}\right) \cdot\left[\mathbf{v}\left(\mathbf{r}_{2}\right)-\mathbf{v}\left(\mathbf{r}_{1}\right)\right]<0 .
$$

The two spheres then progressively rotate around one another, maintaining exact contact for a finite time. This is entirely confirmed by optical observations [1] ; at low $\varphi$, the average time of contact is of the order $2.5 \mathrm{~s}^{-1}$.

Thus there must exist a non-vanishing probability $p$ for one given sphere to be in exact contact with one (or more) other spheres. This $p$ is independent of $s$ and is an increasing function of the volume fraction $\varphi$, going from $p(0)=0$ to $p\left(\varphi_{\mathrm{m}}\right)=1$ (where $\varphi_{\mathrm{m}}$ corresponds to close packing).

When $\varphi$ and $p$ increase, the spheres become progressively associated into clusters. Inside one cluster, any two spheres are linked by at least one path using only nearest neighbour contacts ; these clusters are able to transfer momentum instantaneously, and are thus essential for the mechanical properties; note that the clusters are anisotropic, as shown on figure 1.

\section{Remarks}

a) The clusters have a branched structure. Using a simplified version of eq. (4) (without backflow), we find that one sphere may be in contact for a finite time, simultaneously, with up to eight neighbours. This allows for a significant branching probability, as required for the analogy with percolation $\left(^{1}\right)$.

( $\left.{ }^{1}\right)$ In two dimensions the same criterion would allow only four neighbours : it may be that branching is then very unfrequent, and that the transition does not exist for hard discs.

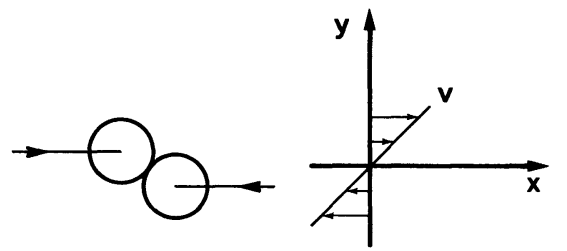

(a)

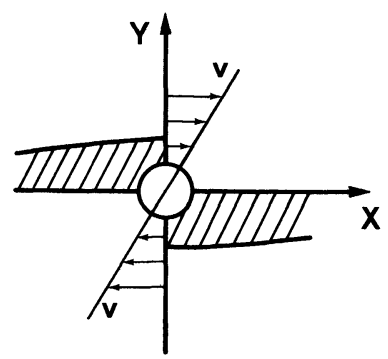

(b)

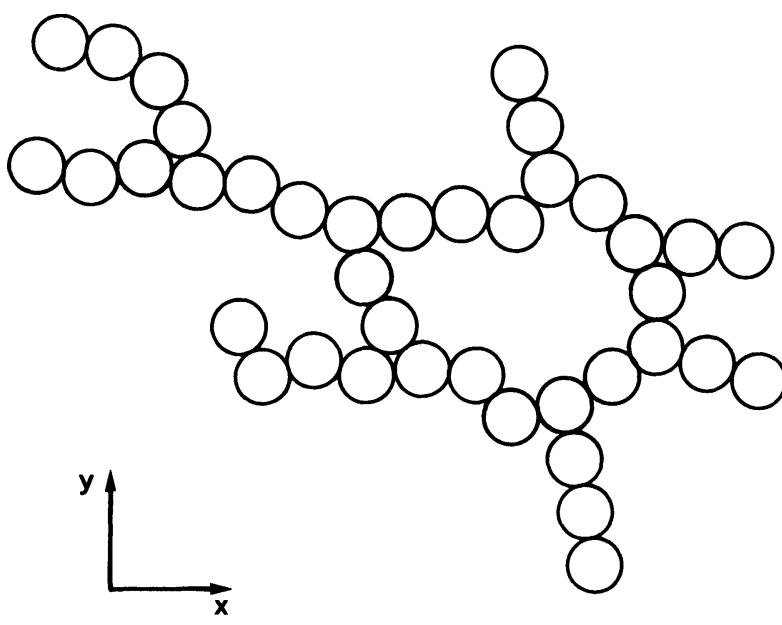

(c)

Fig. 1. - Formation of clusters in simple shear : a) two spheres, driven against each other by the flow, come into contact and then stick against each other for a finite time $; b$ ) regions of approach leading to sticking for sphere $2 ; c$ ) general aspect of a large cluster : note the anisotropy.

b) The clusters are not permanent. While rotating in the ambient fluid, some links are removed, and some others are established. But at any instant, there is a well defined, time independent, distribution of sizes.

c) For the plug flows which we shall consider, in the central region of the tube, the shear gradient is small : we might then ask whether the processes described above for cluster formation have enough time to take place during transit through the tube. However, this does not seem to be a real worry : if we had no clusters, the flow pattern would return 
rapidly to a Poiseuille form, and clusters would be immediately restored.

In the following we assume that the statistics of the clusters is qualitatively similar to what is known for standard (isotropic) percolation problems [7]. We are then led to expect the existence of a critical value $\left(\varphi=\varphi_{\mathrm{c}}\right)$ beyond which there exists one infinite cluster ; for $\varphi$ slightly above $\varphi_{c}\left(\varphi=\varphi_{c}+\Delta \varphi\right)$ there is a certain fraction

$$
\varphi_{\infty}=\text { const. } \Delta \varphi^{\beta_{3}}
$$

of spheres belonging to the infinite cluster. The exponent $\beta_{3}$ may (or may not) coincide with the corresponding exponent for the standard percolation problem $\left(\beta_{p}=0.39\right)$.

3. Hydrodynamics with an infinite cluster. - Since the contact conditions defining a cluster are not maintained by affine transformations, we must assume that the only motions allowed to the infinite cluster are uniform translations and rotations. For the parallel flows of interest here, overall rotations are excluded, and the motion of the infinite cluster is described by a translational velocity parameter $\mathbf{v}_{\mathbf{I}}$ (independent of $r$ ). The rest of the suspension (liquid phase plus finite clusters) has a macroscopic velocity field $\mathbf{v}_{\mathbf{L}}(\mathbf{r})$ ruled by an equation of the form :

$$
-\eta \operatorname{curl} \text { curl } \mathbf{v}_{\mathbf{L}}+\zeta\left(\mathbf{v}_{\mathbf{I}}-\mathbf{v}_{\mathbf{L}}\right)-\nabla p\left(1-\varphi_{\infty}\right)=0
$$

where $\eta$ is an effective viscosity of the fluid part, $\zeta$ a coefficient of mutual friction, and $p$ is the mechanical pressure $\left({ }^{2}\right)$. The last term is the fraction of the pressure gradient acting on the fluid part. We assume that there are no other external forces, acting selectively on the spheres. The condition of zero total force on the infinite cluster imposes :

$$
\int \mathrm{d} \mathbf{r}\left[\zeta\left(\mathbf{v}_{\mathbf{L}}-\mathbf{v}_{\mathbf{I}}\right)-\varphi_{\infty} \nabla p\right]=0 .
$$

Eqs. (6), (7) must be supplemented by conditions at the walls $\left(\mathbf{v}_{\mathrm{L}}=0\right.$ and $\left.\mathbf{v}_{\mathrm{Inormal}}=0\right)$. Typical applications follow.

a) Simple shear between two plates. - The plates are separated by a thickness $D$ of suspension, and move tangentially with opposite velocities $u$ and $-u$. Here $v_{I}=0$, and the velocity profile for $v_{L}$ is of the form :

$$
v_{\mathrm{L}}(y)=u \frac{\sinh (y / \xi)}{\sinh (D / 2 \xi)}
$$

( $\left.{ }^{2}\right)$ The precise form of $\eta$ and $\zeta$ will be discussed in the next section.
(The $y$ axis is normal to the plates and $y=0$ corresponds to the midplane of the slab.) The parameter $\xi$ is called the correlation length, and is defined explicitly by

$$
\xi=(\eta / \zeta)^{1 / 2}
$$

Two limitations of eqs. (6), (8) must be emphasized at this point :

(i) Eq. (6) assumes slow spatial variations and is thus applicable only when $\xi$ is much larger than the interparticle distance. As we shall see, this involves working very near to the percolation threshold.

(ii) Strict application of (6) near the walls requires that the local density of the infinite cluster be unperturbed by the wall. This is not true : percolation clusters are always affected by the proximity of a wall and this effect takes place precisely in the same characteristic length $\xi$. Thus the simple exponential profiles extracted from eq. (6) have only qualitative significance. We shall come back to this point in section 5 .

The apparent viscosity measured in this geometry depends on $D$ and is given by :

$$
\begin{aligned}
\eta_{\mathrm{app}} & =\eta \frac{D}{2 \xi}[\tanh (D / 2 \xi)]^{-1} \\
& \cong \eta \frac{D}{2 \xi} \quad(D \gg \xi) .
\end{aligned}
$$

b) Viscous flow between two fixed plates. - We put the flow lines along $x$, and assume that the flow is driven by a pressure gradient $\partial p / \partial x=p^{\prime}$. Eqs. (6), (7) lead to :

$$
\begin{aligned}
& v_{\mathrm{L}}(y)-v_{\mathrm{I}}=-\zeta^{-1} p^{\prime} \times \\
& \times\left[1-\varphi_{\infty}-\frac{D}{2 \xi} \cosh (y / \xi) / \sinh (D / 2 \xi)\right]
\end{aligned}
$$

and the velocity of the infinite cluster $v_{1}$ is obtained by choosing $y=D / 2$ (since $v_{\mathrm{L}}=0$ at the wall). In the limit $D \gg \xi$ the result is simply

$$
v_{\mathrm{I}}=-\zeta^{-1} p^{\prime} D / 2 \xi \text {. }
$$

Eq. (11) describes a plug flow covering all the slab, except for two boundary layers of thickness $\xi$ near each wall, in qualitative agreement with the data [1]. Note that in the plug region the difference between $v_{\mathrm{L}}$ and $v_{\mathrm{I}}$ is small. On the midplane

$$
\frac{v_{\mathrm{L}}(0)-v_{\mathrm{I}}}{v_{\mathrm{I}}}=\left(1-\varphi_{\infty}\right) \xi / D \quad(D \gg \xi) .
$$

In the observations described in ref. [1] the difference between the liquid velocity $v_{\mathrm{L}}$ and the average velocity of the spheres

$$
v_{\mathrm{s}} \equiv \varphi_{\infty} v_{\mathrm{I}}+\left(1-\varphi_{\infty}\right) v_{\mathrm{L}}
$$

was undetectable. 
4. Scaling laws near threshold. - 1) The correlation length $\xi$ has been defined in eq. (9) but may be qualitatively understood as the size of the largest finite clusters (for any given concentration $\varphi$ ). The percolation analogy leads us to postulate a divergence of $\xi$ for $\varphi$ near $\varphi_{c}$, of the form :

$$
\xi=\xi_{0}\left(\frac{\Delta \varphi}{\varphi_{\mathrm{c}}}\right)^{-v_{3}} .
$$

The length $\xi_{0}$ may depend on the particle radius $(a)$ and on the average interparticle distance $\left(a \varphi^{-1 / 3}\right)$. However, since we are dealing with $\varphi \sim \varphi_{c} \sim 1 / 5$ the distinction between the two is not important, and we may write

$$
\xi=\text { const. } a(\Delta \varphi)^{-v_{3}} .
$$

In the standard percolation problem, we would have $v_{3}=0.85$. It must be emphasized that all our discussion assumes $a \ll \xi$ (for the validity of a continuum description as in eq. (6)) but also $\xi \ll D$. In the opposite limit $(\xi>D)$ the clusters inside the tube become essentially one dimensional, and there is no sharp percolation transition in one dimension.

2) Viscosity of the fluid part $\eta$. - The transport behaviour for standard percolation networks is known to be described well by an effective medium theory, except for a small region near $p_{\mathrm{c}}$ where critical exponents show up. Let us concentrate our attention, however, on this critical region. If the analogy with percolation, or with sol-gel transitions [9] holds, we expect for the parameter $\eta$ a scaling law

$$
\eta / \eta_{0}=\text { const. } \Delta \varphi^{-s_{3}} .
$$

Following the arguments of ref. [8] we might even conjecture $s_{3}$ to coincide with the exponent introduced by Straley [9] for the conductance of a random mixture of normal and superconducting links, $s_{3}=0.7$.

a) For $\varphi<\varphi_{\mathrm{c}}$, the coefficient $\zeta$ is identically equal to zero, and $\eta$ coincides with the measured viscosity of the suspension $\eta_{\text {app }}=\eta$.

b) For $\varphi>\varphi_{c}$, the apparent viscosity of the solution is roughly given by eq. (10), and is different from $\eta$. We shall introduce another conjecture here : namely that the singularity of $\eta$ is described by the same exponent $s_{3}$ above and below threshold. In the regime $D \gg \xi$ (eqs. $(10,14,15))$ would then lead to

$$
\eta_{\text {app }} / \eta_{0}=\text { const. } \frac{D}{a} \Delta \varphi^{\nu_{3}-s_{3}} .
$$

Note that $v_{3}$ may be slightly larger than $s_{3}$, so that $\eta_{\text {app }}$ remains an increasing function of $\varphi$ above threshold. These predictions are summarized in figure 2. The singularity of $\eta_{\mathrm{app}}(\varphi)$ near $\varphi=\varphi_{\mathrm{c}}$ has not been observed to our knowledge ; it may be masked when the ratio $D / s$ is not large enough.

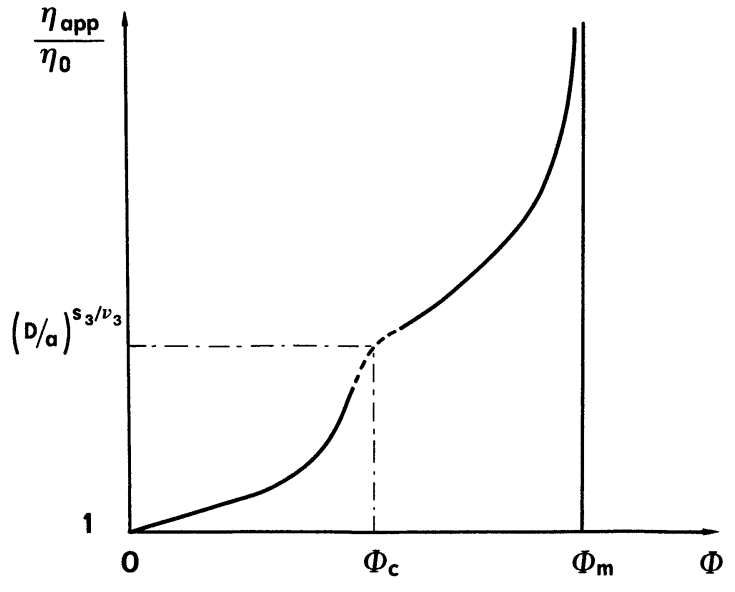

Fig. 2. - Qualitative predictions on the apparent viscosity $\eta_{\text {app }} v s$. sphere concentration. Near the critical concentration there is a small region of rounding due to the finite transverse section $D$ of the flow.

3) The mutual friction coefficient $\zeta$ is derived from eqs. (9), (15) :

$$
\zeta=\eta \zeta^{-2}=\text { const. } \eta_{0} a^{-2}(\Delta \varphi)^{-s_{3}+2 v_{3}} .
$$

Note that in three dimensions $\beta_{3} \neq 2 v_{3}-s_{3}$, and $\zeta$ is not simply proportional to the fraction of spheres belonging to the infinite cluster ; in higher dimensionalities, however, $\zeta$ and $\varphi_{\infty}$ would become proportional, as assumed in ref. [8].

5. Structure of the high shear regions. - The onset of an infinite cluster explains rather naturally the existence of plug flows above a certain critical concentration $\varphi_{\mathrm{c}}$. However, a number of experimental features remain difficult to explain in the simple version of the model which was given above. They are listed below $\left({ }^{3}\right)$.

1) At the critical concentration we would expect an anomaly in the plot of apparent viscosity versus concentration $\left({ }^{4}\right)$, of the type shown in figure 2 . But nothing of this sort has been observed in the existing data.

2) The velocity profiles for plug flow in the regions of strong shear are predicted to be exponential from eq. (6). But the data of Karnis et al. [1] do not give simple exponentials.

3) At fixed concentration we expect the thickness $\xi$ of the sheared layer to scale as $a \Delta \varphi^{-v_{3}}$, i.e. to increase with the sphere radius. The results of ref. [1] go in the opposite direction.

$\left({ }^{3}\right)$ I am indebted to a referee for the objections $(2,3)$.

$\left({ }^{4}\right)$ This anomaly of $\eta(\varphi)$ has nothing to do with the divergence of the viscosity calculated by Bedeaux and Kapral [3] for one particular model (lattice of spheres in the monopole approximation). In our case, direct contact between some of the spheres is essential. 
4) In the regions of strong shear, we expect the velocity of the fluid component to be significantly different from the velocity of the infinite cluster. This is in apparent disagreement with the observations of Karnis et al. [1] who conclude that the velocity of the spheres remains nearly equal to the velocity of the surrounding fluid.

We believe that all these difficulties are related to a reduction of the infinite cluster fraction near the wall. At a distance $x$ from the wall the local fraction $\varphi_{\infty}(x)$ is reduced below the bulk value $\varphi_{\infty}$. Similar effects are known, for instance, in magnetism : near a surface, where each spin benefits only from a reduced number of exchange couplings, the spontaneous magnetization is much smaller than in the bulk [10]. The region of reduced order has a thickness equal to the correlation length $\xi$ (provided that no longrange forces - or, here, backflows - come into play).

Unfortunately most mechanical measurements will be sensitive only to what happens in a thickness $\xi$ near the wall : the region of strong shear is precisely the region where the infinite cluster is weakened. This may allow us to understand qualitatively points $(1,2$, and 4$)$ in the above list. In particular, the velocity of the spheres near the wall should represent predominantly the velocity of finite clusters - equal to that of the solvent.

Thus we conclude that detailed numerical predictions even very near the critical concentration (at large $\xi$ ) must include delicate wall effects : for standard percolation problems, these wall effects are just beginning to be studied [11].

Point (3) in the above list of difficulties is most mysterious : however it must be realized that for the largest diameters studied by Karnis et al. the tube radius corresponds only to five sphere dia- meters : then a number of new effects such as the formation of solid vaults through the tube may become important.

6. Precise checks of the percolation model. - We have seen that the onset of an infinite cluster can provide a natural explanation for plug flows. This idea is not easily compared, however, with the mechanical experiments, which are mainly sensitive to a perturbed layer near the walls. One way to improve the situation would be to use computer calculations (similar to molecular dynamics) for an assembly of spheres. But, on a more experimental basis, it is useful to consider some other methods of proving the existence of an infinite cluster.

1) The most direct method (suggested to us by Dr Clerc) involves using spheres with a conducting surface, and measurement of the electric conductivity of the suspension in simple shear flow. If the same critical concentration $\varphi_{\mathrm{c}}$ is found from the onset of plug flow and from the conductance data, we will know that an infinite cluster is involved.

2) The propagation of longitudinal sound pulses in the suspension might be significantly altered by the presence of the infinite cluster.

3) It might be interesting to try and disrupt the infinite cluster by some external agent (sonication ?) and to look for a resulting removal of plug flows.

Acknowledgments. - This work was initiated in 1975 by conversations with D. Quemada and M. Oddou. More recently, the author has benefited from very stimulating discussions on related subjects with P. Mazur, J. Bedeaux, M. Clerc and E. Guyon, and from helpful comments from two referees.

\section{Notes and references}

[1] Goldsmith, H., MASON, S., in Rheology; theory and application, Eirich ed. (Acad. Press) 1964, vol. 4, p. 85.

Cox, R. G., Mason, S., Ann. Rev. Fluid Mech. 3 (1971) 291.

Karnis, A., Goldsmith, H., Mason, S., J. Colloid Interface Sci. 22 (1966) 531.

[2] See for instance :

KIRKPATRICK, S., Rev. Mod. Phys. 45 (1973) 574.

[3] Bedeaux, D., Kapral, R., Mazur, P., Physica 88A (1977) 88. Bedeaux, D., Kapral, R., Physica (1978).

[4] Our discussion is limited to steady flows in simple shear.
[5] In eq. (4) the velocity $v\left(\mathbf{r}_{i}\right)$ includes the unperturbed flow plus the backflow corrections from all spheres other than $(i)$.

[6] For small $\varphi, p$ grows linearly with $\varphi$.

[7] Stauffer, D., J. Chem. Soc. Faraday Trans. 72 (1976) 1354.

[8] De Gennes, P. G., C. R. Hebd. Séan. Acad. Sci. Paris 286B (1978) 131.

[9] Straley, J. P., Phys. Rev. 15B (1977) 5733.

[10] Fisher, M. E., J. Vac. Sci. Technol. 10 (1973) 665.

[11] Carton, M. and Collectif Marseille (unpublished). 